\title{
p-Summing Operators on Injective Tensor Products of Spaces
}

by

Stephen Montgomery-Smith ${ }^{(*)}$ and Paulette Saab ${ }^{(*)}$

\begin{abstract}
Let $X, Y$ and $Z$ be Banach spaces, and let $\prod_{p}(Y, Z)(1 \leq p<\infty)$ denote the space of $p$-summing operators from $Y$ to $Z$. We show that, if $X$ is a $£_{\infty}$-space, then a bounded linear operator $T: X \hat{\otimes}_{\epsilon} Y \longrightarrow Z$ is 1-summing if and only if a naturally associated operator $T^{\#}: X \longrightarrow \prod_{1}(Y, Z)$ is 1 -summing. This result need not be true if $X$ is not a $£_{\infty}$-space. For $p>1$, several examples are given with $X=C[0,1]$ to show that $T^{\#}$ can be $p$-summing without $T$ being $p$ summing. Indeed, there is an operator $T$ on $C[0,1] \hat{\otimes}_{\epsilon} \ell_{1}$ whose associated operator $T^{\#}$ is 2-summing, but for all $N \in \mathbf{N}$, there exists an $N$-dimensional subspace $U$ of $C[0,1] \hat{\otimes}_{\epsilon} \ell_{1}$ such that $T$ restricted to $U$ is equivalent to the identity operator on $\ell_{\infty}^{N}$. Finally, we show that there is a compact Hausdorff space $K$ and a bounded linear operator $T: C(K) \hat{\otimes}_{\epsilon} \ell_{1} \longrightarrow \ell_{2}$ for which $T^{\#}: C(K) \longrightarrow \prod_{1}\left(\ell_{1}, \ell_{2}\right)$ is not 2-summing.
\end{abstract}

(*) Research supported in part by an NSF Grant DMS 9001796

${ }^{(* *)}$ Research supported in part by an NSF Grant DMS 87500750

A.M.S. (1980) subject classification: 46B99 
Introduction Let $X$ and $Y$ be Banach spaces, and let $X \hat{\otimes}_{\epsilon} Y$ denote their injective tensor product. In this paper, we shall study the behavior of those operators on $X \hat{\otimes}_{\epsilon} Y$ that are $p$-summing.

If $X, Y$ and $Z$ are Banach spaces, then every $p$-summing operator $T: X \hat{\otimes}_{\epsilon} Y \longrightarrow Z$ induces a $p$-summing linear operator $T^{\#}: X \longrightarrow \prod_{p}(Y, Z)$. This raises the following question: given two Banach spaces $Y$ and $Z$, and $1 \leq p<\infty$, for what Banach spaces $X$ is it true that a bounded linear operator $T: X \hat{\otimes}_{\epsilon} Y \longrightarrow Z$ is $p$-summing whenever $T^{\#}: X \longrightarrow \prod_{p}(Y, Z)$ is $p$-summing?

In [11], it was shown that whenever $X=C(\Omega)$ is a space of all continuous functions on a compact Hausdorff space $\Omega$, then $T: C(\Omega) \hat{\otimes}_{\epsilon} Y \longrightarrow Z$ is 1-summing if and only if $T^{\#}: C(\Omega) \longrightarrow \prod_{1}(Y, Z)$ is 1 -summing. We will extend this result by showing that this result still remains true if $X$ is any $£_{\infty}$-space. We will also give an example to show that the result need not be true if $X$ is not a $£_{\infty}$-space. For this, we shall exhibit a 2 -summing operator $T$ on $\ell_{2} \hat{\otimes}_{\epsilon} \ell_{2}$ that is not 1 -summing, but such that the associated operator $T^{\#}$ is 1-summing.

The case $p>1$ turns out to be quite different. Here, the $£_{\infty}$-spaces do not seem to play any important role. We show that for each $1<p<\infty$, there exists a bounded linear operator $T: C[0,1] \hat{\otimes}_{\epsilon} \ell_{2} \longrightarrow \ell_{2}$ such that $T^{\#}: C[0,1] \longrightarrow \prod_{p}\left(\ell_{2}, \ell_{2}\right)$ is $p$-summing, but such that $T$ is not $p$-summing. We will also give an example that shows that, in general, the condition on $T^{\#}$ to be 2 -summing is too weak to imply any good properties for the operator $T$ at all. To illustrate this, we shall exhibit a bounded linear operator $T$ on $C[0,1] \hat{\otimes}_{\epsilon} \ell_{1}$ with values in a certain Banach space $Z$, such that $T^{\#}: C[0,1] \longrightarrow \prod_{2}\left(\ell_{1}, Z\right)$ is 2-summing, but for any given $N \in \mathbf{N}$, there exists a subspace $U$ of $C[0,1] \hat{\otimes}_{\epsilon} \ell_{1}$, with $\operatorname{dim} U=N$, such that $T$ restricted to $U$ is equivalent to the identity operator on $\ell_{\infty}^{N}$.

Finally, we show that there is a compact Hausdorff space $K$ and a bounded linear operator $T: C(K) \hat{\otimes}_{\epsilon} \ell_{1} \longrightarrow \ell_{2}$ for which $T^{\#}: C(K) \longrightarrow \prod_{1}\left(\ell_{1}, \ell_{2}\right)$ is not 2-summing. 


\section{I - Definitions and Preliminaries}

Let $E$ and $F$ be Banach spaces, and let $1 \leq q \leq p<\infty$. An operator $T: E \longrightarrow F$ is said to be $(p, q)$-summing if there exists a constant $C \geq 0$ such that for any finite sequence $e_{1}, e_{2}, \ldots, e_{n}$ in $E$, we have

$$
\left(\sum_{i=1}^{n}\left\|T\left(e_{i}\right)\right\|^{p}\right)^{\frac{1}{p}} \leq C \sup \left\{\left(\sum_{i=1}^{n}\left|e^{*}\left(e_{i}\right)\right|^{q}\right)^{\frac{1}{q}}: e^{*} \in E^{*},\left\|e^{*}\right\| \leq 1\right\} .
$$

We let $\pi_{p, q}(T)$ denote the smallest constant $C$ such that the above inequality holds, and let $\prod_{p, q}(E, F)$ be the space of all $(p, q)$-summing operators from $E$ to $F$ with the norm $\pi_{p, q}$. It is easy to check that $\prod_{p, q}(E, F)$ is a Banach space. In the case $p=q$, we will simply write $\prod_{p}(E, F)$ and $\pi_{p}$. We will use the fact that $T \in \prod_{p, q}(E, F)$ if and only if $\sum_{n}\left\|T e_{n}\right\|^{p}<\infty$ for every infinite sequence $\left(e_{n}\right)$ in $E$ with $\sum_{n}\left|e^{*}\left(e_{n}\right)\right|^{q}<\infty$ for each $e^{*} \in E^{*}$. That is to say, $T$ is in $\prod_{p, q}(E, F)$ if and only if $T$ sends all weakly $\ell_{q}$-summable sequences into strongly $\ell_{p}$-summable sequences. In what follows we shall mainly be interested in the case where $p=q$ and $p=1$ or 2 .

Given two Banach spaces $E$ and $F$, we will let $E \hat{\otimes}_{\epsilon} F$ denote their injective tensor product, that is, the completion of the algebraic tensor product $E \otimes F$ under the cross norm $\|\cdot\|_{\epsilon}$ given by the following formula. If $\sum_{i=1}^{n} e_{i} \otimes x_{i} \in E \otimes F$, then

$$
\left\|\sum_{i=1}^{n} e_{i} \otimes x_{i}\right\|_{\epsilon}=\sup \left\{\left|\sum_{i=1}^{n} e^{*}\left(e_{i}\right) x^{*}\left(x_{i}\right)\right|:\left\|e^{*}\right\| \leq 1,\left\|x^{*}\right\| \leq 1, e^{*} \in E^{*}, x^{*} \in F^{*}\right\} .
$$

We will say that a bounded linear operator $T$ between two Banach spaces $E$ and $F$ is called an integral operator if the bilinear form $\tau$ defines an element of $\left(E \hat{\otimes}_{\epsilon} F^{*}\right)^{*}$, where $\tau$ is induced by $T$ according to the formula $\tau\left(e, x^{*}\right)=x^{*}(T e)\left(e \in E, x^{*} \in F^{*}\right)$. We will define the integral norm of $T$, denoted by $\|T\|_{\text {int }}$, by

$$
\|T\|_{\mathrm{int}}=\sup \left\{\left|\sum_{i=1}^{n} x_{i}^{*}\left(T e_{i}\right)\right|:\left\|\sum_{i=1}^{n} e_{i} \otimes x_{i}^{*}\right\|_{\epsilon} \leq 1\right\} .
$$


The space of all integral operators from a Banach space $E$ into a Banach space $F$ will be denoted by $I(E, F)$. We note that $I(E, F)$ is a Banach space under the integral norm \|\|$_{\text {int }}$.

We will say that a Banach space $X$ is a $£_{\infty}$-space if, for some $\lambda>1$, we have that for every finite dimensional subspace $B$ of $X$, there exists a finite dimensional subspace $E$ of $X$ containing $B$, and an invertible bounded linear operator $T: E \longrightarrow \ell_{\infty}^{\operatorname{dim}} E$ such that $\|T\|\left\|T^{-1}\right\| \leq \lambda$.

It is well known that for any Banach spaces $E$ and $F$, if $T$ is in $I(E, F)$, then it is also in $\prod_{1}(E, F)$, with $\pi_{1}(T) \leq\|T\|_{\text {int }}$. But $I(E, F)$ is strictly included in $\prod_{1}(E, F)$. It was shown in $[12, \mathrm{p} .477]$ that a Banach space $E$ is a $£_{\infty}$-space if and only if for any Banach space $F$, we have that $I(E, F)=\prod_{1}(E, F)$. We will use this characterization of $£_{\infty}$-spaces in the sequel.

Finally, we note the following characterization of 1-summing operators (called right semi-integral by Grothendieck in [5]), which will be used later.

Proposition 1 Let $E$ and $F$ be Banach spaces. Then the following properties about a bounded linear operator $T$ from $E$ to $F$ are equivalent:

(i) $T$ is 1-summing;

(ii) There exists a Banach space $F_{1}$, and an isometric injection $\varphi: F \longrightarrow F_{1}$, such that $\varphi \circ T: E \longrightarrow F_{1}$ is an integral operator.

For all other undefined notions we shall refer the reader to either [3], [7] or [10]. 


\section{1-Summing and Integral Operators}

Let $X$ and $Y$ be Banach spaces with injective tensor product $X \hat{\otimes}_{\epsilon} Y$. For a Banach space $Z$, any bounded linear operator $T: X \hat{\otimes}_{\epsilon} Y \longrightarrow Z$ induces a linear operator $T^{\#}$ on $X$ by

$$
T^{\#} x(y)=T(x \otimes y) \quad(y \in Y) .
$$

It is clear that the range of $T^{\#}$ is the space $£(Y, Z)$ of bounded linear operators from $Y$ into $Z$, and that $T^{\#}$ is a bounded linear operator.

In this section, we are going to investigate the 1-summing operators, and the integral operators, on $X \hat{\otimes}_{\epsilon} Y$. We will use Proposition 1 to relate these two ideas together. First of all, we have the following result.

Theorem 2 Let $X, Y$ and $Z$ be Banach spaces, and let $T: X \hat{\otimes}_{\epsilon} Y \longrightarrow Z$ be a bounded linear operator. Denote by $i: Z \longrightarrow Z^{* *}$ the isometric embedding of $Z$ into $Z^{* *}$. Then the following two properties are equivalent:

(i) $T \in I\left(X \hat{\otimes}_{\epsilon} Y, Z\right)$;

(ii) $\hat{i} \circ T \in I\left(X, I\left(Y, Z^{* *}\right)\right)$, where $\hat{i}: I(Y, Z) \longrightarrow I\left(Y, Z^{* *}\right)$ is defined by $\hat{i}(U)=i \circ U$ for each $U \in I(Y, Z)$.

In particular, if $T^{\#} \in I(X, I(Y, Z))$, then $T \in I\left(X \hat{\otimes}_{\epsilon} Y, Z\right)$.

Proof: First, we show that $\left(X \hat{\otimes}_{\epsilon} Y\right) \hat{\otimes}_{\epsilon} Z^{*}$ and $X \hat{\otimes}_{\epsilon}\left(Y \hat{\otimes}_{\epsilon} Z^{*}\right)$ are isometrically isomorphic to one another. To see this, note that the algebraic tensor product is an associative operation, that is, $(X \otimes Y) \otimes Z^{*}$ and $X \otimes\left(Y \otimes Z^{*}\right)$ are algebraically isomorphic. Also, they are both generated by elements of the form $\sum_{i=1}^{n} x_{i} \otimes y_{i} \otimes z_{i}^{*}$, where $x_{i} \in X, y_{i} \in Y$ and $z_{i}^{*} \in Z^{*}$. Now, if we let $B\left(X^{*}\right), B\left(Y^{*}\right)$ and $B\left(Z^{* *}\right)$ denote the dual unit balls of $X^{*}, Y^{*}$ and $Z^{* *}$ equipped with their respective weak* topologies, then the spaces $\left(X \otimes_{\epsilon} Y\right) \otimes_{\epsilon} Z^{*}$ and $X \otimes_{\epsilon}\left(Y \otimes_{\epsilon} Z^{*}\right)$ embed isometrically into $C\left(B\left(X^{*}\right) \times B\left(Y^{*}\right) \times B\left(Z^{* *}\right)\right)$ in a natural way, by

$$
\left\langle\sum_{i=1}^{n} x_{i} \otimes y_{i} \otimes z_{i}^{*}, \quad\left(x^{*}, y^{*}, z^{* *}\right)\right\rangle=\sum_{i=1}^{n} x^{*}\left(x_{i}\right) y^{*}\left(y_{i}\right) z^{* *}\left(z_{i}^{*}\right),
$$


where $\sum_{i=1}^{n} x_{i} \otimes y_{i} \otimes z_{i}^{*}$ is in $\left(X \otimes_{\epsilon} Y\right) \otimes_{\epsilon} Z^{*}$ or $X \otimes_{\epsilon}\left(Y \otimes_{\epsilon} Z^{*}\right)$, and $\left(x^{*}, y^{*}, z^{* *}\right)$ is in the compact set $B\left(X^{*}\right) \times B\left(Y^{*}\right) \times B\left(Z^{* *}\right)$. Thus both spaces $\left(X \hat{\otimes}_{\epsilon} Y\right) \hat{\otimes}_{\epsilon} Z^{*}$ and $X \hat{\otimes}_{\epsilon}\left(Y \hat{\otimes}_{\epsilon} Z^{*}\right)$ can be thought of as the closure in $C\left(B\left(X^{*}\right) \times B\left(Y^{*}\right) \times B\left(Z^{* *}\right)\right)$ of the algebraic tensor product of $X, Y$ and $Z^{*}$.

Now let us assume that $T: X \hat{\otimes}_{\epsilon} Y \longrightarrow Z$ is an integral operator. Then the bilinear map $\tau$ on $X \hat{\otimes}_{\epsilon} Y \times Z^{*}$, given by $\tau\left(u, z^{*}\right)=z^{*}(T u)$ for $u \in X \hat{\otimes}_{\epsilon} Y$ and $z^{*} \in Z^{*}$, defines an element of $\left(X \hat{\otimes}_{\epsilon} Y \hat{\otimes}_{\epsilon} Z^{*}\right)^{*}$, that is,

$$
\|T\|_{\text {int }}=\sup \left\{\mid \sum_{i=1}^{n} z_{i}^{*}\left(T\left(x_{i} \otimes y_{i}\right)\right):\left\|\sum_{i=1}^{n} x_{i} \otimes y_{i} \otimes z_{i}^{*}\right\|_{\epsilon} \leq 1\right\} .
$$

To show that for every $x$ in $X$ the operator $T^{\#} x$ is in $I(Y, Z)$, with

$$
\left\|T^{\#} x\right\|_{\text {int }} \leq\|x\|\|T\|_{\text {int }}
$$

is easy. This is because, for each $x \in X$, the operator $T^{\#} x$ is the composition of $T$ with the bounded linear operator from $Y$ to $X \hat{\otimes}_{\epsilon} Y$, which to each $y$ in $Y$ gives the element $x \otimes y$.

If $i: Z \longrightarrow Z^{* *}$ denotes the isometric embedding of $Z$ into $Z^{* *}$, it induces a bounded linear operator $\hat{i}: I(Y, Z) \longrightarrow I\left(Y, Z^{* *}\right)$ given by $\hat{i}(U)=i \circ U$ for all $U \in I(Y, Z)$. It is immediate that $\hat{i}$ is an isometry. We will now show that the operator $\hat{i} \circ T^{\#}$ : $X \longrightarrow I\left(Y, Z^{* *}\right)$ is integral. It is well known (see $\left[3\right.$, p. 237]) that the space $I\left(Y, Z^{* *}\right)$ is isometrically isomorphic to the dual space $\left(Y \hat{\otimes}_{\epsilon} Z^{*}\right)^{*}$. Thus to show that $\hat{i} \circ T^{\#}$ : $X \longrightarrow\left(Y \hat{\otimes}_{\epsilon} Z^{*}\right)^{*}$ is an integral operator, we need to show that it induces an element of $\left(X \hat{\otimes}_{\epsilon}\left(Y \hat{\otimes}_{\epsilon} Z^{*}\right)\right)^{*}$. For this, it is enough to note that, by our discussion concerning the isometry of $\left(X \hat{\otimes}_{\epsilon} Y\right) \hat{\otimes}_{\epsilon} Z^{*}$ and $X \hat{\otimes}_{\epsilon}\left(Y \hat{\otimes}_{\epsilon} Z^{*}\right)$, that

$$
\left\|\hat{i} \circ T^{\#}\right\|_{\text {int }}=\sup \left\{\left|\sum_{i=1}^{n} \hat{i} \circ T^{\#} x_{i}, y_{i} \otimes z_{i}^{*}\right|:\left\|\sum_{i=1}^{n} x_{i} \otimes y_{i} \otimes z_{i}^{*}\right\|_{\epsilon} \leq 1\right\}
$$


But for each $x \in X, y \in Y$ and $z^{*} \in Z^{*}$, we have

$$
\left\langle\hat{i} \circ T^{\#} x, y \otimes z^{*}\right\rangle=\left\langle T(x \otimes y), z^{*}\right\rangle .
$$

Hence, from $(*)$ and $\left({ }^{* *}\right)$, it follows that

$$
\|\hat{i} \circ T\|_{\text {int }}=\|T\|_{\text {int }} .
$$

Thus we have shown that (i) $\Rightarrow$ (ii). The proof of (ii) $\Rightarrow$ (i) follows in a similar way. If $\hat{i} \circ T^{\#}: X \longrightarrow I\left(Y, Z^{* *}\right)$ is an integral operator, then one can show that $i \circ T: X \hat{\otimes}_{\epsilon} Y \longrightarrow$ $Z^{* *}$ is integral, which in turn implies that $T$ itself is integral (see [3, p. 233]).

Finally, the last assertion follows easily, since if $T^{\#}: X \longrightarrow I(Y, Z)$ is integral, then $\hat{i} \circ T$ is integral (see [3, p. 232]).

Since the mapping $\hat{i}: I(Y, Z) \longrightarrow I\left(Y, Z^{* *}\right)$ is an isometry, Proposition 1 coupled with Theorem 2 implies that, if $T: X \hat{\otimes}_{\epsilon} Y \longrightarrow Z$ is an integral operator, then $T^{\#}$ : $X \longrightarrow I(Y, Z)$ is 1-summing. This result can be shown directly from the definitions. In what follows we shall present a sketch of that alternative approach.

Theorem 3 Let $X, Y$ and $Z$ be Banach spaces, and let $T: X \hat{\otimes}_{\epsilon} Y \longrightarrow Z$ be a bounded linear operator. If $T$ is integral, then $T^{\#}: X \longrightarrow I(Y, Z)$ is 1 -summing. If in addition $X$ is a $£_{\infty}$-space, then $T: X \hat{\otimes}_{\epsilon} Y \longrightarrow Z$ is integral if and only if $T^{\#}: X \longrightarrow I(Y, Z)$ is integral.

Proof: First, we will show that, if $T: X \hat{\otimes}_{\epsilon} Y \longrightarrow Z$ is an integral operator, then $T^{\#}$ is in $\prod_{1}(X, I(Y, Z))$ with $\pi_{1}\left(T^{\#}\right) \leq\|T\|_{\text {int }}$. Let $x_{1}, x_{2}, \ldots, x_{n}$ be in $X$, and fix $\epsilon>0$. For each $i \leq n$, there exists $n_{i} \in \mathbf{N},\left(y_{i j}\right)_{j \leq n_{i}}$ in $Y$, and $\left(z_{i j}^{*}\right)_{j \leq n_{i}}$ in $Z^{*}$, such that $\left\|\sum_{j=1}^{n i} y_{i j} \otimes z_{i j}^{*}\right\|_{\epsilon} \leq 1$, and

$$
\left\|T^{\#} x_{i}\right\|_{\text {int }} \leq \sum_{j=1}^{n_{i}} z_{i j}^{*}\left(T\left(x_{i} \otimes y_{i j}\right)\right)+\frac{\epsilon}{2^{i}} .
$$


Since $T$ is an integral operator, and

$$
\left\|\sum_{i=1}^{n} \sum_{j=1}^{n_{i}} x_{i} \otimes y_{i j} \otimes z_{i j}^{*}\right\|_{\epsilon} \leq \sup \left\{\sum_{i=1}^{n}\left|x^{*}\left(x_{i}\right)\right|:\left\|x^{*}\right\| \leq 1, x^{*} \in X^{*}\right\},
$$

it follows that

$$
\sum_{i=1}^{n} \sum_{j=1}^{n_{i}} z_{i j}^{*}\left(T\left(x_{i} \otimes y_{i j}\right)\right) \leq\|T\|_{\text {int }} \sup \left\{\sum_{i=1}^{n}\left|x^{*}\left(x_{i}\right)\right|:\left\|x^{*}\right\| \leq 1, x^{*} \in X^{*}\right\} .
$$

Therefore

$$
\sum_{i=1}^{n}\left\|T^{\#} x_{i}\right\|_{\text {int }} \leq\|T\|_{\text {int }} \sup \left\{\sum_{i=1}^{n}\left|x^{*}\left(x_{i}\right)\right|: x^{*} \in X^{*},\left\|x^{*}\right\| \leq 1\right\}+\epsilon .
$$

Now, if in addition $X$ is a $£_{\infty}$-space, then by [12, p. 477], the operator $T^{\#}$ is indeed integral.

Remark 4 If $X=C(\Omega)$ is a space of continuous functions defined on a compact Hausdorff space $\Omega$, one can deduce a similar result to Theorem 3 from the main result of [13].

Our next result extends a result of [16] to $£_{\infty}$-spaces, where it was shown that whenever $X=C(\Omega)$, a space of all continuous functions on a compact Hausdorff space $\Omega$, then a bounded linear operator $T: C(\Omega) \hat{\otimes}_{\epsilon} Y \longrightarrow Z$ is 1-summing if and only if $T^{\#}: C(\Omega) \longrightarrow \prod_{1}(Y, Z)$ is 1-summing. This also extends a result of [14] where similar conclusions were shown to be true for $X=A(K)$, a space of continuous affine functions on a Choquet simplex $K$ (see [2]).

We note that one implication follows with no restriction on $X$. If $X, Y$ and $Z$ are Banach spaces, and $T: X \hat{\otimes}_{\epsilon} Y \longrightarrow Z$ is a 1-summing operator, then $T^{\#}$ takes its values in $\prod_{1}(Y, Z)$. This follows from the fact that for each $x \in X$, the operator $T^{\#} x$ is the composition of $T$ with the bounded linear operator from $Y$ into $X \hat{\otimes}_{\epsilon} Y$ which to each $y$ in $Y$ gives the element $x \otimes y$ in $X \hat{\otimes}_{\epsilon} Y$, and hence

$$
\pi_{1}\left(T^{\#} x\right) \leq\|x\| \pi_{1}(T)
$$


Moreover, one can proceed as in [16] to show that $T^{\#}: X \longrightarrow \prod_{1}(Y, Z)$ is 1 -summing.

Theorem 5 If $X$ is a $£_{\infty}$ space, then for any Banach spaces $Y$ and $Z$, a bounded linear operator $T: X \hat{\otimes}_{\epsilon} Y \longrightarrow Z$ is 1-summing if and only if $T^{\#}: X \longrightarrow \prod_{1}(Y, Z)$ is 1 -summing.

Proof: Let $T: X \hat{\otimes}_{\epsilon} Y \longrightarrow Z$ be such that $T^{\#}: X \longrightarrow \prod_{1}(Y, Z)$ is 1 -summing. Since $X$ is a $£_{\infty}$-space, it follows from $\left[14\right.$, p. 477] that $T^{\#}: X \longrightarrow \prod_{1}(Y, Z)$ is an integral operator. Let $\varphi$ denote the isometric embedding of $Z$ into $C\left(B\left(Z^{*}\right)\right)$, the space of all continuous scaler functions on the unit ball $B\left(Z^{*}\right)$ of $Z^{*}$ with its weak*-topology. This induces an isometry

$$
\begin{gathered}
\hat{\varphi}: \prod_{1}(Y, Z) \longrightarrow \prod_{1}\left(\left(Y, C\left(B\left(Z^{*}\right)\right)\right),\right. \\
\hat{\varphi}(U)=\varphi \circ U
\end{gathered}
$$

Now, it follows from $\left[15\right.$, p. 301], that $\prod_{1}\left(Y, C\left(B\left(Z^{*}\right)\right)\right)$ is isometric to $I\left(Y, C\left(B\left(Z^{*}\right)\right)\right)$. Hence we may assume that $\hat{\varphi} \circ T^{\#}: X \longrightarrow I\left(Y, C\left(B\left(Z^{*}\right)\right)\right)$ is an integral operator. Moreover, it is easy to check that $(\varphi \circ T)^{\#}=\hat{\varphi} \circ T^{\#}$. By Theorem 2 the operator $\varphi \circ T: X \hat{\otimes}_{\epsilon} Y \longrightarrow C\left(B\left(Z^{*}\right)\right)$ is an integral operator, and hence $T$ is in $\prod_{1}\left(X \hat{\otimes}_{\epsilon} Y, Z\right)$ by Proposition 1.

In the following section we shall, among other things, exhibit an example that illustrates that it is crucial for the space $X$ to be a $£_{\infty}$-space if the conclusion of Theorem 5 is to be valid.

\section{2-summing Operators and some Counter-examples.}

In this section we shall study the behavior of 2-summing operators on injective tensor product spaces. As we shall soon see, the behavior of such operators when $p=2$ is quite different from when $p=1$. For instance, unlike the case $p=1$, the $£_{\infty}$-spaces don't seem to play any particular role. In fact, we shall exhibit operators $T$ on $C[0,1] \hat{\otimes}_{\epsilon} \ell_{2}$ which are not 2-summing, yet their corresponding operators $T^{\#}$ are. We will also give other interesting examples that answer some other natural questions. 
We will present the next theorem for $p=2$, but the same result is true for any $1 \leq p<\infty$, with only minor changes.

Theorem 6 Let $X, Y$ and $Z$ be Banach spaces. If $T: X \hat{\otimes}_{\epsilon} Y \longrightarrow Z$ is a 2 -summing operator, then $T^{\#}: X \longrightarrow \prod_{2}(Y, Z)$ is a 2 -summing operator.

Proof: If $T: X \hat{\otimes}_{\epsilon} Y \longrightarrow Z$ is 2-summing, then using the same kind of arguments that we have given above, it can easily be shown that for each $x \in X$, that $T^{\#} x \in \prod_{2}(Y, Z)$, with $\pi_{2}\left(T^{\#} x\right) \leq \pi_{2}(T)\|x\|$.

Now we will show that $T^{\#}: X \longrightarrow \prod_{2}(Y, Z)$ is 2-summing. Let $\left(x_{n}\right)$ be in $X$ such that $\sum_{n}\left|x^{*}\left(x_{n}\right)\right|^{2}<\infty$ for each $x^{*}$ in $X^{*}$. Fix $\epsilon>0$. For each $n \geq 1$, let $\left(y_{n m}\right)$ be a sequence in $Y$ such that

$$
\sup \left\{\left(\sum_{m=1}^{\infty}\left|y^{*}\left(y_{n m}\right)\right|^{2}\right)^{1 / 2}:\left\|y^{*}\right\| \leq 1, y^{*} \in Y^{*}\right\} \leq 1,
$$

and

$$
\pi_{2}\left(T^{\#} x_{n}\right) \leq\left(\sum_{m=1}^{\infty}\left\|T\left(x_{n} \otimes y_{n m}\right)\right\|^{2}\right)^{1 / 2}+\frac{\epsilon}{2^{n}}
$$

Then

$$
\left[\pi_{2}\left(T^{\#} x_{n}\right)\right]^{2} \leq \sum_{m=1}^{\infty}\left\|T\left(x_{n} \otimes y_{n m}\right)\right\|^{2}+\frac{\epsilon}{2^{n-1}}\left(\sum_{m=1}^{\infty}\left\|T\left(x_{n} \otimes y_{n m}\right)\right\|^{2}\right)^{1 / 2}+\frac{\epsilon^{2}}{2^{2 n}} .
$$

Now, consider the sequence $\left(x_{n} \otimes y_{n m}\right)$ in $X \hat{\otimes}_{\epsilon} Y$. For each $\xi \in\left(X \hat{\otimes}_{\epsilon} Y\right)^{*} \simeq I\left(X, Y^{*}\right)$ we have that

$$
\begin{aligned}
\sum_{m, n}\left|\xi\left(x_{n}\right)\left(y_{n m}\right)\right|^{2} & =\sum_{n=1}^{\infty} \sum_{m=1}^{\infty}\left|\xi\left(x_{n}\right)\left(y_{n m}\right)\right|^{2} \\
& \leq \sum_{n=1}^{\infty}\left\|\xi\left(x_{n}\right)\right\|^{2}
\end{aligned}
$$

Since $\xi \in I\left(X, Y^{*}\right)$, it follows that $\xi \in \prod_{2}\left(X, Y^{*}\right)$, and so

$$
\sum_{n=1}^{\infty}\left\|\xi\left(x_{n}\right)\right\|^{2}<\infty
$$


Hence we have shown that for all $\xi \in\left(X \hat{\otimes}_{\epsilon} Y\right)^{*}$,

$$
\sum_{m, n}\left|\xi\left(x_{n}\right)\left(y_{n m}\right)\right|^{2}<\infty
$$

Since $T \in \prod_{2}\left(X \hat{\otimes}_{\epsilon} Y, Z\right)$, we have that

$$
\sum_{m, n}\left\|T\left(x_{n} \otimes y_{n m}\right)\right\|^{2}<\infty
$$

and therefore

$$
\sum_{n}\left[\pi_{2}\left(T^{*^{*}} x_{n}\right)\right]^{2}<\infty
$$

Remark 7 The above result extends a result of [1], where it was shown that if $T$ : $X \hat{\otimes}_{\epsilon} Y \longrightarrow Z$ is $p$-summing for $1 \leq p<\infty$, then $T^{\#}: X \longrightarrow £(Y, Z)$ is $p$-summing.

Now we shall give the example that we promised at the end of section II.

Theorem 8 There exists a bounded linear operator $T: \ell_{2} \hat{\otimes}_{\epsilon} \ell_{2} \longrightarrow \ell_{2}$ such that $T$ is not 1 -summing, yet $T^{\#}: \ell_{2} \longrightarrow \pi_{1}\left(\ell_{2}, \ell_{2}\right)$ is 1 -summing.

Proof: First, we note the well known fact that $\ell_{2} \hat{\otimes}_{\epsilon} \ell_{2}=\mathcal{K}\left(\ell_{2}, \ell_{2}\right)$, the space of all compact operators from $\ell_{2}$ to $\ell_{2}$. Now we define $T$ as the composition of two operators.

Let $P: \mathcal{K}\left(\ell_{2}, \ell_{2}\right) \longrightarrow c_{0}$ be the operator defined so that for each $K \in \mathcal{K}\left(\ell_{2}, \ell_{2}\right)$,

$$
P(K)=\left(K\left(e_{n}\right)\left(e_{n}\right)\right)
$$

where $\left(e_{n}\right)$ is the standard basis of $\ell_{2}$. It is well known [10, p.145] that the sequence $\left(e_{n} \otimes e_{n}\right)$ in $\ell_{2} \hat{\otimes}_{\epsilon} \ell_{2}$ is equivalent to the $c_{0}$-basis, and that the operator $P$ defines a bounded linear projection of $\mathcal{K}\left(\ell_{2}, \ell_{2}\right)$ onto $c_{0}$.

Let $S: c_{0} \longrightarrow \ell_{2}$ be the bounded linear operator such that for each $\left(\alpha_{n}\right) \in c_{0}$

$$
S\left(\alpha_{n}\right)=\left(\frac{\alpha_{n}}{n}\right)
$$


It is easily checked $[7$, p. 39] that $S$ is a 2 -summing operator that is not 1 -summing.

Now we define $T: \mathcal{K}\left(\ell_{2}, \ell_{2}\right) \longrightarrow \ell_{2}$ to be $T=S \circ P$. Thus $T$ is 2 -summing but not 1-summing. It follows from Theorem 6 that the induced operator $T^{\#}: \ell_{2} \longrightarrow \prod_{2}\left(\ell_{2}, \ell_{2}\right)$ is 2 -summing. Since $\ell_{2}$ is of cotype 2 , it follows from [10, p. 62], that for any Banach space $E$, we have $\prod_{2}\left(\ell_{2}, E\right)=\prod_{1}\left(\ell_{2}, E\right)$, and that there exists a constant $C>0$ such that for all $U \in \prod_{2}\left(\ell_{2}, E\right)$ we have

$$
\pi_{1}(U) \leq C \pi_{2}(U)
$$

This implies that $T^{\#}$ is 1 -summing as an operator taking its values in $\prod_{1}\left(\ell_{2}, \ell_{2}\right)$.

Remark 9 We do not need to use Theorem 6 to show that $T^{\#}$ is 1 -summing in the example above. Instead, we can use the following argument. First note that $T^{\#}$ factors as follows:

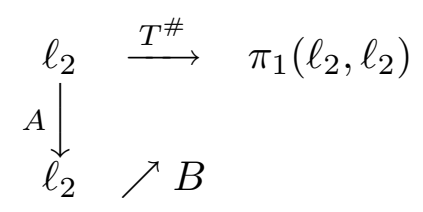

Here $A: \ell_{2} \rightarrow \ell_{2}$ is the 1 -summing operator defined by

$$
A\left(\alpha_{n}\right)=\left(\frac{\alpha_{n}}{n}\right)
$$

for each $\left(\alpha_{n}\right) \in \ell_{2}$, and $B: \ell_{2} \longrightarrow \pi_{1}\left(\ell_{2}, \ell_{2}\right)$ is the natural embedding of $\ell_{2}$ into the space $\pi_{1}\left(\ell_{2}, \ell_{2}\right)$ defined by

$$
B\left(\beta_{n}\right)\left(\gamma_{n}\right)=\left(\beta_{n} \gamma_{n}\right)
$$

for each $\left(\beta_{n}\right),\left(\gamma_{n}\right) \in \ell_{2}$.

Now we will give two examples concerning the case when $p>1$. We will show that we do not have a converse to Theorem 8 , even when the underlying space $X$ is a $£_{\infty}$-space. 
First, let us fix some notation. In what follows we shall denote the space $\ell_{p}(\mathbf{Z})$ by $\ell_{p}$, and call its standard basis $\left\{e_{n}: n \in \mathbf{Z}\right\}$. Thus if $x=(x(n)) \in \ell_{p}$, then $x(n)=\left\langle x, e_{n}\right\rangle$, and

$$
\left.\|x\|_{\ell_{p}}=\left(\sum_{n=1}^{\infty}\left|\left\langle x, e_{n}\right\rangle\right|^{p}\right\rangle\right)^{\frac{1}{p}} .
$$

For $f \in L_{p}[0,1]$, we let

$$
\|f\|_{L_{p}}=\left(\int_{0}^{1}|f(t)|^{p} d t\right)^{\frac{1}{p}} .
$$

If $\Omega$ is a compact Hausdorff space, and $Y$ is a Banach space, then $C(\Omega, Y)=C(\Omega) \hat{\otimes}_{\epsilon} Y$ will denote the Banach space of continuous $Y$-valued functions on $\Omega$ under the supremum norm.

We recall that since $\ell_{2}$ is of cotype 2 , we have that $\prod_{2}\left(\ell_{2}, \ell_{2}\right)=\prod_{1}\left(\ell_{2}, \ell_{2}\right)$. We also recall that, if $u=\sum_{n=1}^{\infty} \alpha_{n} e_{n} \otimes e_{n}$ is a diagonal operator in $\prod_{2}\left(\ell_{2}, \ell_{2}\right)$, then

$$
\pi_{2}(u)=\left(\sum_{n=1}^{\infty}\left|\alpha_{n}\right|^{2}\right)^{\frac{1}{2}}=\text { the Hilbert-Schmidt norm of } u .
$$

Theorem 10 For each $1<p<\infty$, there is a bounded linear operator $T: C\left([0,1], \ell_{2}\right) \rightarrow \ell_{2}$ that is not $p$-summing, but such that $T^{\#}: C[0,1] \longrightarrow \Pi_{p}\left(\ell_{2}, \ell_{2}\right)$ is $p$-summing.

Proof: We present the proof for $p \leq 2$. The case where $p>2$ follows by the same argument. For each $n \in \mathbf{Z}$, let $\epsilon_{n}(t):[0,1] \rightarrow \mathbf{C}, \epsilon_{n}(t)=e^{2 \pi \text { int }}$ denote the standard trigonometric basis of $L_{2}[0,1]$. If $f \in L_{1}[0,1]$, let $\hat{f}(n)=\int_{0}^{1} f(t) \epsilon_{n}(t) d t$ denote the usual Fourier coefficient of $f$. For each $\lambda=\left(\lambda_{n}\right)$, where $\left|\lambda_{n}\right| \leq 1$ for all $n \in \mathbf{Z}$, define the operator

$$
T_{\lambda}: C\left([0,1], \ell_{2}\right) \longrightarrow \ell_{2}
$$

such that for $\varphi \in C\left([0,1], \ell_{2}\right)$ we have

$$
T_{\lambda} \varphi=\left(\lambda_{n}\left\langle\hat{\varphi}(n), e_{n}\right\rangle\right) .
$$


Here $\hat{\varphi}(n)=$ Bochner $-\int_{0}^{1} \varphi(t) \epsilon_{n}(t) d t$.

The operator $T_{\lambda}$ is a bounded linear operator, with $\left\|T_{\lambda} \varphi\right\|_{\ell_{2}} \leq\|\varphi\|$. To see this, note that for $\varphi \in C\left([0,1], \ell_{2}\right)$ we have

$$
\begin{aligned}
\left\|T_{\lambda} \varphi\right\|_{\ell_{2}}^{2} & =\sum_{n}\left|\lambda_{n}\right|^{2}\left|\left\langle\hat{\varphi}(n), e_{n}\right\rangle\right|^{2} \\
& \leq \sum_{n}\left|\left\langle\hat{\varphi}(n), e_{n}\right\rangle\right|^{2} \\
& \leq \sum_{n} \int_{0}^{1}\left|\left\langle\varphi(t), e_{n}\right\rangle\right|^{2} d t \\
& =\int_{0}^{1}\|\varphi(t)\|_{\ell_{2}}^{2} d t \\
& \leq \sup _{t}\|\varphi(t)\|_{\ell_{2}}^{2} .
\end{aligned}
$$

Now, note that if $f \in C([0,1])$, and $x \in \ell_{2}$, then

$$
T_{\lambda}(f \otimes x)=\left(\lambda_{n} \hat{f}(n)\left\langle x, e_{n}\right\rangle\right),
$$

and hence the operator $T_{\lambda}^{\#}: C[0,1] \rightarrow £\left(\ell_{2}, \ell_{2}\right)$ is such that

$$
T_{\lambda}^{\#} f(x)=\left(\lambda_{n} \hat{f}(n)\left\langle x, e_{n}\right\rangle\right)
$$

Thus

$$
\pi_{2}\left(T_{\lambda}^{\#} f\right)=\left(\sum_{n}\left|\lambda_{n}\right|^{2}|\hat{f}(n)|^{2}\right)^{\frac{1}{2}} .
$$

Hence, by Hölder's inequality,

$$
\pi_{2}\left(T_{\lambda}^{\#} f\right) \leq\left\|\left(\lambda_{n}\right)\right\|_{\ell_{r}}\|(\hat{f}(n))\|_{\ell_{q}},
$$

where $\frac{1}{r}+\frac{1}{q}=\frac{1}{2}$. By the Hausdorff-Young inequality, we have that

$$
\|(\hat{f}(n))\|_{\ell_{q}} \leq\|f\|_{L_{p}},
$$

where $1 \leq p \leq 2$ and $\frac{1}{p}+\frac{1}{q}=1$. Thus

$$
\pi_{2}\left(T_{\lambda}^{\#} f\right) \leq\left\|\left(\lambda_{n}\right)\right\|_{\ell_{r}}\|f\|_{L_{p}},
$$


for $1 \leq p \leq 2,2 \leq r \leq \infty$ and $\frac{1}{p}=\frac{1}{r}+\frac{1}{2}$. This shows that if $\left\|\left(\lambda_{n}\right)\right\|_{\ell_{r}}<\infty$, then

(1) $T_{\lambda}^{\#}(C[0,1]) \subseteq \pi_{2}\left(\ell_{2}, \ell_{2}\right)=\pi_{p}\left(\ell_{2}, \ell_{2}\right)$;

(2) $T_{\lambda}^{\#}: C[0,1] \longrightarrow \pi_{p}\left(\ell_{2}, \ell_{2}\right)$ is $p$-summing.

Now, let $U \subset C\left([0,1], \ell_{2}\right)$ be the closed linear span of $\left\{\epsilon_{i} \otimes e_{i}, a_{i} \in \mathbf{Z}\right\}$. Then $U$ is isometrically isomorphic to $\ell_{2}$. This is because

$$
\begin{aligned}
\left\|\sum_{i} \mu_{i} \epsilon_{i} \otimes e_{i}\right\| & =\sup _{t \in[0,1]}\left\|\left(\mu_{n} \epsilon_{n}(t)\right)\right\|_{\ell_{2}} \\
& =\left\|\left(\mu_{i} \epsilon_{i}\left(t_{0}\right)\right)\right\|_{\ell_{2}},
\end{aligned}
$$

for some $t_{0} \in[0,1]$, and hence

$$
\left\|\sum_{i} \mu_{i} \epsilon_{i} \otimes e_{i}\right\|=\left(\sum_{i}\left|\mu_{i}\right|^{2}\right)^{\frac{1}{2}} .
$$

Moreover

$$
T_{\lambda}\left(\epsilon_{i} \otimes e_{i}\right)=\lambda_{i} e_{i} \quad \text { for all } i \in \mathbf{Z},
$$

Therefore, we have the following commuting diagram

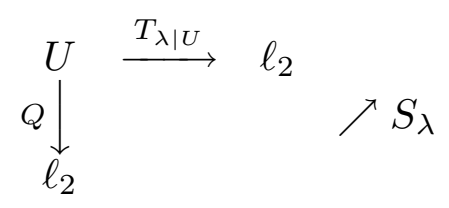

where $Q: U \rightarrow \ell_{2}$ is the isomorphism from $U$ onto $\ell_{2}$ such that $Q\left(\epsilon_{n} \otimes e_{n}\right)=e_{n}$ for all $n \in \mathbf{Z}$, and $S_{\lambda}: \ell_{2} \longrightarrow \ell_{2}$ is the operator given by $S_{\lambda}\left(e_{n}\right)=\lambda_{n} e_{n}$. So to show that $T_{\lambda}$ is not $p$-summing, it is sufficient to show that one can pick $\lambda=\left(\lambda_{n}\right)$ such that $S_{\lambda}$ is not $p$-summing. To do this, we consider two cases. If $p=2$, we take $\lambda_{n}=1$ for all $n \in \mathbf{Z}$. Then the map $S_{\lambda}$ induced on $\ell_{2}$ is the identity map which is not $s$-summing for any $s<\infty$. If $1<p<2$, let $\lambda_{n}=\frac{1}{|n+1|^{\frac{1}{r}} \log |n+1|}$, so that $\left\|\left(\lambda_{n}\right)\right\|_{\ell_{r}}<\infty$. Then the map $S_{\lambda}: \ell_{2} \longrightarrow \ell_{2}$ is not $s$-summing for any $s<r$. To show this, we may assume, without loss of generality, that $s \geq 2$. Let $x_{n}=e_{n}$ for all $n \geq 1$, and note that

$$
\sup _{x^{*} \in B\left(\ell_{2}\right)}\left(\sum_{n}\left|x^{*}\left(x_{n}\right)\right|^{s}\right)^{\frac{1}{s}} \leq\left\|x^{*}\right\|_{\ell_{2}} \leq 1,
$$


whilst

$$
\left(\sum_{n}\left\|\lambda_{n} x_{n}\right\|^{s}\right)^{\frac{1}{s}}=\infty
$$

While the operators $T_{\lambda}$ in the previous example failed to be $p$-summing, they were all $(2,1)$-summing. This suggests the following question: suppose $T: C([0,1], Y) \longrightarrow Z$ is a bounded linear operator such that $T^{\#}: C[0,1] \longrightarrow \prod_{2}(Y, Z)$ is 2 -summing. What can we say about $T$ ? Is $T(2,1)$-summing? The following example shows that $T$ can be very bad.

Theorem 11 There exists a Banach space $Z$, and a bounded linear operator $T: C\left([0,1], \ell_{1}\right) \rightarrow Z$ such that $T^{\#}: C[0,1] \rightarrow \prod_{2}\left(\ell_{1}, Z\right)$ is 2-summing, with the property that, for any $N \in \mathbf{N}$, there exists a subspace $U$ of $C\left([0,1], \ell_{1}\right)$ with $\operatorname{dim} U=N$, such that $T$ restricted to $U$ behaves like the identity operator on $\ell_{\infty}^{N}$. In particular $T$ is not $(2,1)$-summing.

Proof: If $X$ and $Y$ are Banach spaces, we denote by $X \hat{\otimes}_{\pi} Y$ the projective tensor product, that is, the completion of the algebraic tensor product of $X$ and $Y$ under the norm

$$
\|u\|_{\pi}=\inf \left\{\sum_{i=1}^{n}\left\|x_{i}\right\|\left\|y_{i}\right\|, u=\sum_{i=1}^{n} x_{i} \otimes y_{i}\right\} .
$$

It is well known that $\left(X \hat{\otimes}_{\pi} Y\right)^{*}$ is isometrically isomorphic to the space $£\left(X, Y^{*}\right)$ of all bounded linear operators from $X$ to $Y^{*}$.

Let $Z=C\left([0,1], \ell_{1}\right)+L_{2}[0,1] \hat{\otimes}_{\pi} \ell_{2}$ be the Banach space with the norm

$$
\|x\|_{Z}=\inf \left\{\left\|x^{\prime}\right\|_{\epsilon}+\left\|x^{\prime \prime}\right\|_{\pi}: x=x^{\prime}+x^{\prime \prime}\right\}
$$

where \|\|$_{\epsilon}$ denotes the sup norm in $C\left([0,1], \ell_{1}\right)$, and \|\|$_{\pi}$ denotes the norm of the projective tensor product $L_{2}[0,1] \hat{\otimes}_{\pi} \ell_{2}$. Let

$$
T: C\left([0,1], \ell_{1}\right) \longrightarrow Z
$$


be the identity operator.

We first see that for each $f \in C[0,1]$, the operator $T^{\#} f: \ell_{1} \rightarrow Z$ is 2 -summing with

$$
\pi_{2}\left(T^{\#} f\right) \leq \pi_{2}(I)\left\|T^{\#} f\right\|_{£\left(\ell_{2}, Z\right)},
$$

where $I: \ell_{1} \longrightarrow \ell_{2}$ is the natural mapping. This is because, for each $f \in C[0,1]$, and each $x \in \ell_{1}$, we have that

$$
\|T(f \otimes x)\| \leq\|f \otimes x\|_{L_{2} \hat{\otimes}_{\pi} \ell_{2}} \leq\|f\|_{L_{2}}\|x\|_{\ell_{2}} .
$$

To see that $T^{\#}: C[0,1] \longrightarrow \prod_{2}\left(\ell_{1}, X\right)$ is 2 -summing, note that $\left\|T^{\#} f\right\|_{£\left(\ell_{2}, Z\right)} \leq\|f\|_{L_{2}}$, and hence if $f_{1}, \ldots, f_{n} \in C[0,1]$, then

$$
\begin{aligned}
\left(\sum_{k=1}^{n}\left[\pi_{2}\left(T^{\#} f_{k}\right)\right]^{2}\right)^{\frac{1}{2}} & \leq \pi_{2}(I)\left(\sum_{k=1}^{n}\left\|f_{k}\right\|_{L_{2}}^{2}\right)^{\frac{1}{2}} \\
& \leq \pi_{2}(I) \pi_{2}(J) \sup _{t \in[0,1]}\left\|\left(\sum_{K=1}^{n}\left|f_{k}(t)\right|^{2}\right)^{\frac{1}{2}}\right\|
\end{aligned}
$$

Here $J: C[0,1] \longrightarrow L_{2}[0,1]$ denotes the natural mapping.

Now we define the space $U$, a closed linear subspace of $C\left([0,1], \ell_{1}\right)$. Let $\left\{f_{i j}: 1 \leq\right.$ $i, j \leq N\}$ be disjoint functions in $C[0,1]$, for which $0 \leq f_{i j} \leq 1,\left\|f_{i j}\right\|=1$, each $f_{i j}$ is supported in an interval of length $\frac{1}{N^{2}}$, and

$$
\int_{0}^{1} f_{i j} d t=\frac{1}{2 N^{2}} \text { and } \int_{0}^{1} f_{i j}^{2} d t=\frac{1}{3 N^{2}} .
$$

Let $\left\{e_{i j}: 1 \leq i, j \leq N\right\}$ be distinct unit vectors in $\ell_{1}$. We let $U=\left\{\sum_{i, j} \lambda_{i} f_{i j} \otimes e_{i j}, \lambda_{i} \in \mathbf{R}\right\}$.

Now we consider $T$ restricted to $U$. If $\sum_{i, j} \lambda_{i} f_{i j} \otimes e_{i j} \in U$, then

$$
\left\|\sum_{i, j} \lambda_{i} f_{i j} \otimes e_{i j}\right\|_{\epsilon} \leq \sup _{i}\left|\lambda_{i}\right|
$$

and hence

$$
\left\|\sum_{i, j} \lambda_{i} f_{i j} \otimes e_{i j}\right\|_{Z} \leq \sup _{i}\left|\lambda_{i}\right|
$$


Let $y_{i}^{*}=N \sum_{j} f_{i j} \otimes e_{i j}$, and set $x=\sum_{i, j} \lambda_{i} f_{i j} \otimes e_{i j}$. Then whenever $x=x^{\prime}+x^{\prime \prime}$, with $x^{\prime} \in C\left([0,1], \ell_{1}\right)$ and $x^{\prime \prime} \in L_{2}[0,1] \hat{\otimes}_{\pi} \ell_{2}$, we know that

$$
\left|y_{i}^{*}(x)\right| \leq\left|y_{i}^{*}\left(x^{\prime}\right)\right|+\left|y_{i}^{*}\left(x^{\prime \prime}\right)\right|
$$

Hence

$$
\left|y_{i}^{*}(x)\right| \leq\left\|y_{i}^{*}\right\|_{C\left([0,1], \ell_{1}\right)^{*}}\left\|x^{\prime}\right\|_{\epsilon}+\left\|y_{i}^{*}\right\|_{\left(L_{2}[0,1] \hat{\otimes}_{\pi} \ell_{2}\right)^{*}}\left\|x^{\prime \prime}\right\|_{\pi} .
$$

But

$$
\begin{aligned}
\left\|y_{i}^{*}\right\|_{C\left([0,1], \ell_{1}\right)^{*}} & =N \sum_{i=1}^{N} \int_{\operatorname{supp} f_{i j}}\left|f_{i j}\right| d t \\
& =N \cdot \frac{N}{2 N^{2}}=\frac{1}{2}
\end{aligned}
$$

and, since $\left(L_{2}[0,1] \hat{\otimes}_{\pi} \ell_{2}\right)^{*}$ is isometric to $£\left(L_{2}[0,1], \ell_{2}\right)$,

$$
\begin{aligned}
\left\|y_{i}^{*}\right\|_{\left(L_{2}[0,1] \hat{\otimes}_{\pi} \ell_{2}\right)^{*}} & =\sup \left\{\left[\sum_{j=1}^{N}\left(N \int_{0}^{1} f_{i j} g d t\right)^{2}\right]^{\frac{1}{2}}:\|g\|_{L_{2}} \leq 1\right\} \\
& \leq \sup \left\{N\left[\sum_{j=1}^{N} \int_{0}^{1} f_{i j}^{2} d t \cdot \int_{\operatorname{supp} f_{i j}}|g|^{2} d t\right]^{\frac{1}{2}}:\|g\|_{L_{2}} \leq 1\right\} \\
& =\frac{1}{\sqrt{3}}\left\{\left(\sum_{j=1}^{N} \int_{\operatorname{supp} f_{i j}}|g|^{2} d t\right)^{\frac{1}{2}}:\|g\|_{2} \leq 1\right\} \\
& =\frac{1}{\sqrt{3}} .
\end{aligned}
$$

Therefore

$$
\left|y_{i}^{*}(x)\right| \leq \frac{1}{2}\left\|x^{\prime}\right\|_{\epsilon}+\frac{1}{\sqrt{3}}\left\|x^{\prime \prime}\right\|_{\pi}, \leq \frac{1}{\sqrt{3}}\|x\|
$$

However,

$$
\begin{aligned}
y_{i}^{*}(x) & =N \sum_{j=1}^{N} \lambda_{i} \int_{0}^{1} f_{i j}^{2} d t \\
& =N^{2} \lambda_{i} \frac{1}{3 N^{2}}=\frac{\lambda_{i}}{3} .
\end{aligned}
$$

Therefore

$$
\begin{aligned}
\left\|\sum_{i, j} \lambda_{i} f_{i j} \otimes e_{i j}\right\|_{Z} & \geq \sqrt{3} \sup _{i}\left|y_{i}^{*}(x)\right| \\
& \geq \frac{1}{\sqrt{3}} \sup \left|\lambda_{i}\right| .
\end{aligned}
$$


Thus the space $U$ is isomorphic to $\ell_{\infty}^{N}$, and we have the commuting diagram

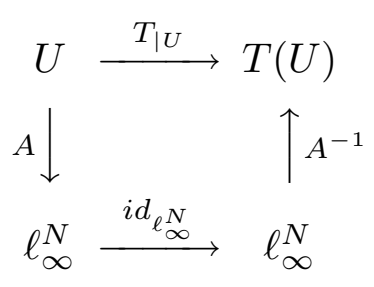

where $A: U \rightarrow \ell_{\infty}^{N}$ is the isomorphism between $U$ and $\ell_{\infty}^{N}$.

\section{Operators that factor through a Hilbert space}

It is well known that $£\left(X, \ell_{2}\right)=\prod_{2}\left(X, \ell_{2}\right)$ whenever $X$ is $C(K)$ or $\ell_{1}$. One might ask whether this is true when $X=C\left(K, \ell_{1}\right)$. Indeed one could ask the weaker question: if $T: C\left(K, \ell_{1}\right) \longrightarrow \ell_{2}$ is bounded, does it follow that the induced operator $T^{\#}$ is 2 -summing? We answer this question in the negative.

Theorem 12 There is a compact Hausdorff space $K$ and a bounded linear operator $T: C\left(K, \ell_{1}\right) \longrightarrow \ell_{2}$ for which $T^{\#}: C(K) \longrightarrow \prod_{1}\left(\ell_{1}, \ell_{2}\right)$ is not 2 -summing.

Proof: First, we show that there is a compact Hausdorff space $K$, and an operator $R: C(K) \longrightarrow \ell_{\infty}$ that is $(2,1)$-summing but not 2 -summing. To see this, let $K=[0,1]$, and consider the natural embedding $C[0,1] \longrightarrow L_{2,1}[0,1]$, where $L_{2,1}[0,1]$ is the Lorentz space on $[0,1]$ with the Lebesque measure (see [6]). By [11], it follows that this map is $(2,1)$-summing. To show that this map is not 2-summing, we argue in a similar fashion to [8]. For $n \in \mathbf{N}$, consider the functions $e_{i}(t)=f\left(t+\frac{1}{i} \bmod 1\right)(1 \leq i \leq n)$, where $f(t)=\frac{1}{\sqrt{t}}$ if $t \geq \frac{1}{n}$ and $\sqrt{n}$ otherwise. Then it is an easy matter to verify that for some constant $C>0$,

$$
\left(\sum_{i=1}^{n}\left|e^{*}\left(e_{i}\right)\right|^{2}\right)^{\frac{1}{2}} \leq C \sqrt{\log n}
$$

for every $e^{*}$ in the unit ball of $C[0,1]^{*}$, whereas

$$
\left(\sum_{i=1}^{n}\left\|e_{i}\right\|_{L_{2,1}[0,1]}^{2}\right)^{\frac{1}{2}} \geq C^{-1} \log n .
$$


Finally, since $L_{2,1}[0,1]$ is separable, it embeds isometrically into $\ell_{\infty}$.

Define $T: C\left(K, \ell_{1}\right) \rightarrow \ell_{2}$ as follows: for $\varphi=\left(f_{n}\right) \in C\left(K, \ell_{1}\right)$, let

$$
T\left(f_{n}\right)=\sum_{n} R f_{n}(n) e_{n}
$$

Then $T$ is bounded, for

$$
\begin{aligned}
\left\|T\left(f_{n}\right)\right\|_{2} & =\left(\sum_{n}\left|R f_{n}(n)\right|^{2}\right)^{\frac{1}{2}} \\
& \leq\left(\sum_{n}\left\|R f_{n}\right\|_{\ell_{\infty}}^{2}\right)^{\frac{1}{2}} \\
& \leq \pi_{2,1}(R) \sup _{t \in K} \sum_{n}\left|f_{n}(t)\right| .
\end{aligned}
$$

Thus

$$
\|T\| \leq \pi_{2,1}(R)
$$

But $T^{\#}: C(K) \longrightarrow £\left(\ell_{1}, \ell_{2}\right)$ is not 2 -summing, because for each $f \in C(K)$, the operator $T^{\#} f: \ell_{1} \longrightarrow \ell_{2}$ is the diagonal operator $\sum_{n} R f(n) e_{n} \otimes e_{n}$. Hence the strong operator norm of $T^{\#} f$ is

$$
\left\|T^{\#} f\right\|=\sup _{n}|R f(n)|=\|R f\|_{\ell_{\infty}} .
$$

Thus $T^{\#}: C(K) \longrightarrow £\left(\ell_{1}, \ell_{2}\right)$ is not 2 -summing, because $R: C(K) \longrightarrow \ell_{\infty}$ is not 2-summing.

\section{Discussions and concluding remarks}

Remark 13 Theorem 12 shows that if $X$ and $Y$ are Banach spaces such that $£\left(X, \ell_{2}\right)=$ $\prod_{2}\left(X, \ell_{2}\right)$ and $£\left(Y, \ell_{2}\right)=\prod_{2}\left(X, \ell_{2}\right)$, then $X \hat{\otimes}_{\epsilon} Y$ need not share this property. This observation could also be deduced from arguments presented in [4] (use Example 3.5 and the proof of Proposition 3.6 to show that there is a bounded operator $T:\left(\ell_{1} \oplus \ell_{1} \oplus \ldots \oplus\right.$ $\left.\ell_{1}\right)_{\ell_{\infty}} \longrightarrow \ell_{2}$ that is not $p$-summing for any $p<\infty$ ). 
Remark 14 In the proof of Theorem 2 we showed that the injective tensor product is an associative operation, that is, if $X, Y$ and $Z$ are Banach spaces, then $\left(X \hat{\otimes}_{\epsilon} Y\right) \hat{\otimes}_{\epsilon} Z$ is isometrically isomorphic to $X \hat{\otimes}_{\epsilon}\left(Y \hat{\otimes}_{\epsilon} Z\right)$. It is not hard to see that the same is true for the projective tensor product. However, we can conclude from Theorem 12 that what is known as the $\gamma_{2}^{*}$-tensor product is not an associative operation.

If $E$ and $F$ are Banach spaces, and $T: E \longrightarrow F$ is a bounded linear operator, following [10], we say that $T$ factors through a Hilbert space if there is a Hilbert space $H$, and operators $B: E \longrightarrow H$ and $A: H \longrightarrow F$ such that $T=A \circ B$. We let $\gamma_{2}(T)=\inf \{\|A\|\|B\|\}$, where the infimum runs over all possible factorization of $T$, and denote the space of all operators $T: E \longrightarrow F$ that factor through a Hilbert space by $\Gamma_{2}(E, F)$. It is not hard to check that $\gamma_{2}$ defines a norm on $\Gamma_{2}(E, F)$, making $\Gamma_{2}(E, F)$ a Banach space. We define the $\gamma_{2}^{*}$-norm $\|\quad\|_{*}$ on $E \otimes F$ (see [9] or [10]) in which the dual of $E \otimes F$ is identified with $\Gamma_{2}\left(E, F^{*}\right)$, and let $E \hat{\otimes}_{\gamma_{2}^{*}} F$ denote the completion of $\left(E \otimes F,\|\quad\|_{*}\right)$.

The operator $T: C(K) \hat{\otimes}_{\gamma_{2}^{*}} \ell_{1} \longrightarrow \ell_{2}$ exhibited in Theorem 12, induces a bounded linear functional on $\left[\left(C(K) \hat{\otimes}_{\gamma_{2}^{*}} \ell_{1}\right) \hat{\otimes}_{\gamma_{2}^{*}} \ell_{2}\right]^{*}$. Now we see that if $C(K) \hat{\otimes}_{\gamma_{2}^{*}}\left(\ell_{1} \hat{\otimes}_{\gamma_{2}^{*}} \ell_{2}\right)$ were isometrically isomorphic to $\left(C(K) \hat{\otimes}_{\gamma_{2}^{*}} \ell_{1}\right) \hat{\otimes}_{\gamma_{2}^{*}} \ell_{2}$, then the operator $T^{\#}: C(K) \rightarrow £\left(\ell_{1}, \ell_{2}\right)$ would induce a bounded linear functional on $\left[C(K) \hat{\otimes}_{\gamma_{2}^{*}}\left(\ell_{1} \hat{\otimes}_{\gamma_{2}^{*}} \ell_{2}\right)\right]^{*}$, showing that $T^{\#} \in$ $\Gamma_{2}\left(C(K), £\left(\ell_{1}, \ell_{2}\right)\right)$, implying that $T^{\#}$ would be 2 -summing [10, p. 62]. This contradiction shows that $C(K) \hat{\otimes}_{\gamma_{2}^{*}}\left(\ell_{1} \hat{\otimes}_{\gamma_{2}^{*}} \ell_{2}\right)$ and $\left(C(K) \hat{\otimes}_{\gamma_{2}^{*}} \ell_{1}\right) \hat{\otimes}_{\gamma_{2}^{*}} \ell_{2}$ cannot be isometrically isomorphic.

Another example showing that the $\gamma_{2}^{*}$-tensor product is not associative was given by Pisier (private communication). 


\section{Bibliography}

[1] R. Bilyeu, and P. Lewis, Some Mapping Properties of Representing Measures, Ann. Math Pure Appl. CIX (1976) p. 273-287.

[2] G. Choquet, Lectures on Analysis, Vol. II, Benjamin, New York, (1969).

[3] J. Diestel, and J.J. Uhl Jr., Vector Measures, Math Surveys, 15, AMS, Providence, RI (1977).

[4] T. Figiel, J. Lindenstrauss, and V. Milman, The dimension of almost spherical sections of convex bodies, Acta Mathematica, 139, (1977), p. 53-94.

[5] A. Grothendick, Produits tensoriels topologiques et espaces nucléaires, Mem. A.M.S. 16, (1955).

[6] R.A. Hunt, On L(p,q) spaces, L'Enseignement Math. (2), 12, (1966), p. 249-275.

[7] G.J.O. Jameson, Summing and Nuclear Norms in Banach Space Theory, LMSST 8, Cambridge University Press (1987).

[8] G.J.O. Jameson, Relations between summing norms of mappings on $\ell_{\infty}$, Math. Z, 194, (1987), p. 89-94.

[9] S. Kwapien, On operators factorizable through $L_{p}$-spaces, Bull. Soc. Math. France, Mém 31-32, (1972), p. 215-225.

[10] G. Pisier, Factorization of Linear Operators and Geometry of Banach Spaces, AMS CBMS 60, Providence RI (1986).

[11] G. Pisier, Factorization of operators through $L_{p \infty}$ or $L_{p 1}$ and non commutative generalizations, Math. Ann., 276, (1986), p. 105-136.

[12] J.R. Retherford, and C. Stegall, Fully Nuclear and Completely Nuclear Operators with applications to $£_{1}$ and $£_{\infty}$ spaces, T.A.M.S., 163, (1972) p. 457-492.

[13] P. Saab, Integral Operators on Spaces of Continuous Vector Valued Functions, Proc. Amer. Math. Soc. (to appear).

[14] B. Smith, Some Bounded Linear Operators On the Spaces $C(\Omega, E)$ and $A(K, E)$, Ph.D. Dissertation, The University of Missouri-Columbia, 1989. 
[15] C. Stegall, Characterization of Banach spaces whose duals are $L_{1}$ spaces, Is. J. of Math, 11, (1972) p. 299-308.

[16] C. Swartz, Absolutely summing and dominated operators on spaces of vector-valued continuous functions, T.A.M.S., 179, (1973) p. 123-132.

University of Missouri

Dept. of Math.

Columbia, MO 65211 